\section{Do you want to sell your practice?}

With the property market booming, now is a better time than ever to sell your practice. But where to begin?

With decades of industry experience and a finger on the pulse of the current financial market, the team at practices4dentists is able to offer highquality, real-time advice that is directly tailored to the unique needs of your practice.

The team manages every aspect of the dental practice sale process, from valuation and marketing to practice visits and completion of sale agreements.

So, if you're thinking of selling, enlist the help of the experts at practices4dentists today.

For more information call 0845345 5060, email info@4dentistsgroup.com or visit www.4dentistsgroup.com.

\section{Augment your digital workflow}

Augment your digital workflow with the EXTARO 300 dental microscope. It offers multiple breakthrough visualisation modes designed to support dental caries detection and facilitate more simplified restorative treatment.

The EXTARO 300 also features an integrated HD camera that can record images and video footage wirelessly to the ZEISS Connect App, which can then be directly transferred to your local network.

This visual information can be used to better educate patients about their condition and highlight areas that require treatment, promoting more informed decision-making. Clinicians can also benefit from mitigating litigious risks using documented evidence of the treatment process.

For more information call Nuview on 01453 872266, email info@nuview-ltd.com or visit www.nuview.co.uk.

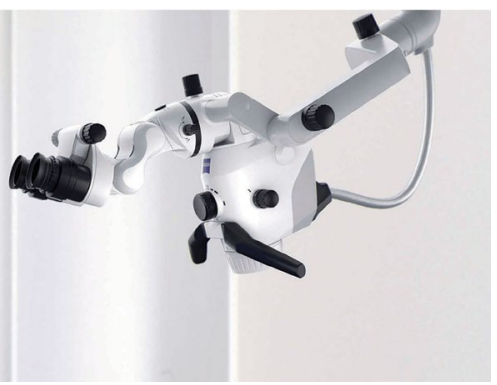

\section{Future-proof your practice team}

The recruitment challenges that practices are currently facing as a result of COVID19 , Brexit and regulatory reforms have led to practice management nationwide being put under a great deal of pressure, particularly with the surge of patients post-lockdown. These unknowns may be out of your control but redirecting your focus to what can be actively managed will enable you to retain and attract valuable team members despite the state of circumstances going into the new year.

Software of Excellence want to provide you with solutions that will promote a harmonious and happy working environment - introducing a toolkit of Dentist Portal and MyPractice Cloud Pay (MPC Pay) will allow for just this.

Empowering team members with their own data will provide your practice with a degree of transparency that will leave your staff feeling valued throughout their journey with you, without having to lose control of the 'important stuff'.

Integrating seamlessly with Dentist Portal, MPC Pay provides greater clarity for associates in terms of their income and performance - allowing them to be better informed over their contribution to the practice and to take ownership of discussions surrounding it. From clear and concise breakdowns of their earnings/deductions to KPI summaries and invoicing, individuals are able to review, forecast and enhance their performance on the go like never before.

Uncovering information in this way not only reveals opportunities for your team members but also for the overall success of your practice.

Growing your practice no longer relies solely on the quality of dental care you provide, but stems from the foundations of a great practice team. This is why building strong staff relationships is now absolutely crucial, and with the technology now to support this, trust within a team can be built from the very first instance.

The team is the most important asset a dental practice has and, as leaders, dentists and managers should strive to show personal commitment to their teams and find new ways to inspire confidence in the future. Empowering, encouraging and listening to your staff will help boost their morale, and whilst doing so isn't an obligation, with external pressures now at play, it's time to act.

Talk to the Software of Excellence team today about how you can drive individual and practice performance with Dentist Portal and MPC Pay - call 01634266800.

For more information visit: www. softwareofexcellence.com.

\title{
Financial flexibility for dental patients
}

Openpay, the UK's next generation, interest-free, buy now, pay smarter payment solution, has announced its largest expansion to date into the UK healthcare market, signing partnerships with 93 dental practices - 45 are already live, with a further 18 due to go live at the time of writing.

The partner practices are found throughout the UK, reaching a diverse collection of consumers in need of financial flexibility for dental work.

Openpay's proposition provides payment plans with no interest or hidden fees, crafted to put Brits' health, and financial wellbeing, first. Openpay will be offering all payment plans in-practice, meaning patients will have an immediate solution to spread the cost, rather than pay the entire bill upfront.

Openpay Group Ltd's 'Buy now pay later' (BNPL) payment solution delivers the most flexible plans in the market over a payment period of up to nine months in the UK. Following an initial payment at the time of purchase, customers can spread payments into equal monthly instalments and can align future payments with their pay cycle, helping them to manage their finances more easily. Customers pay no interest, ever, and there are no hidden fees.

For more information visit www. openpay.co.uk. 